\title{
Edge dislocations bowing out from a row of collinear obstacles in $\mathrm{Al}$
}

\author{
Shuozhi $\mathrm{Xu}^{\mathrm{a}, *}$, Liming Xiong ${ }^{\mathrm{b}}$, Youping Chen ${ }^{\mathrm{c}}$, David L. McDowell ${ }^{\mathrm{a}, \mathrm{d}}$ \\ ${ }^{a} G W W$ School of Mechanical Engineering, Georgia Institute of Technology, Atlanta, GA \\ 30332-0405, USA \\ ${ }^{b}$ Department of Aerospace Engineering, Iowa State University, Ames, IA 50011, USA \\ ${ }^{c}$ Department of Mechanical and Aerospace Engineering, University of Florida, \\ Gainesville, FL 32611-6250, USA \\ ${ }^{d}$ School of Materials Science and Engineering, Georgia Institute of Technology, Atlanta, \\ GA 30332-0245, USA
}

\begin{abstract}
The bowing of edge dislocations from a row of collinear obstacles in $\mathrm{Al}$ is studied using concurrent atomistic-continuum simulations of submicron-sized realizations containing up to 238 million atoms. Results show that (1) as the number of adjacent bowed-out dislocation segments increases, the critical dislocation depinning stress approaches that for an infinite array of obstacles and (2) for the unstable overall semi-elliptic dislocation configuration, the presence of intermediate obstacles reduces the dislocation half-loop height, but doesn't affect the critical shear stress. Our work highlights the significance of the effects of adjacent bowed-out segments on cooperative dislocation bow-out.
\end{abstract}

Keywords: Concurrent atomistic-continuum method, aluminum, dislocation theory, plastic deformation, simulation

\footnotetext{
${ }^{*}$ Corresponding author

Email address: shuozhixu@gatech.edu (Shuozhi Xu)

Preprint submitted to Scripta Materialia

June 15, 2016
}

(C) 2016. This manuscript version is made available under the Elsevier user license http://www.elsevier.com/open-access/userlicense/1.0/ 
Dislocation bow-out between pinning points is central to dislocation/obstacle interactions in crystalline materials [1]. Investigating the detailed process of dislocations bowing out and moving through a field of obstacles in metals aids in understanding the onset of flow through lattice, work hardening, and dislocation multiplication from Frank-Read sources [2]. Several modelling techniques, including molecular dynamics (MD) [3, 4], discrete dislocation (DD) $[5,6]$, phase field method $[7,8]$, and level set method [9], have been employed to simulate dislocation bow-out. In most of these studies, only one dislocation segment bows out from an isolated pair of obstacles. In reality, obstacles are distributed randomly in pure metals and alloys, with an average spacing $L$ of about $30 \sim 300 \mathrm{~nm}[10,11]$. Therefore, it is important to account for the effects of distributed bowed-out segments in modeling a given dislocation bow-out, in a way that considers both medium and long range elastic interactions as well as dislocation core effects.

Traditional dislocation bow-out analysis is based on dislocation line tension theory. Continuum dislocation theory predicts that the effective line tension of a curved dislocation segment is a logarithmic function of its initial distance $L$ between two neighboring obstacles, namely, $\ln L$ [12]. In the presence of adjacent dislocation segments, however, the line tension of a given dislocation segment is altered [13]. Specifically, the negative interaction energy of a dislocation and its adjacent bowed-out segments reduces its line tension and hence lowers the critical stress required to bypass the obstacles [14]. This effect is particularly important for a dislocation line lying in a single slip plane and pinned by regularly spaced obstacles [14]. Simulations based on the dislocation self-stress show that, because of their opposite Burgers vec- 
tor, the dipole-like attractive force between dislocation branches terminating on a given obstacle results in a "pinching" effect and facilitates dislocation bow-out $[15,16]$. The role of adjacent dislocation segments becomes more important with a decreasing $D / L$, where $D$ is the obstacle diameter [17]. In dislocation/obstacle interactions, dislocations that depin from adjacent obstacles aid in pulling the branches together and diminish the strengthening effect [18].

Continuum models for dislocation/obstacle interactions apply to the dislocation bow-out process if the dislocation bypasses the obstacles following the Orowan bypass mechanism [19]. Most of these models assume that an infinitely long dislocation bypasses an infinite array of equally spaced obstacles. For obstacles with a finite size $D$, the term $\ln L$ is replaced by $\ln \bar{D}$ by taking into account of the interactions between three closest dislocation branches, where $\bar{D}=\left(D^{-1}+L^{-1}\right)^{-1}$ [17]. Most simulations employ periodic boundary conditions (PBCs) along the dislocation line direction. In dislocation/precipitate interactions, Bacon et al. [17] included three precipitates in one simulation supercell with PBCs to represent an infinite row. Crone et al. [20] found that introducing additional voids beyond three doesn't show a significant influence on the critical depinning stress in dislocation/void interactions. Studies of a dislocation passing a random array of obstacles have been pursued using line tension models [21-23] and DD simulations [24] . Nevertheless, the effects of bow-out of other dislocation segments on the critical shear stress of a given dislocation bow-out in an image-free model have not been explored quantitatively, to the best of our knowledge.

In this paper, we perform quasistatic concurrent atomistic-continuum 
$(\mathrm{CAC})$ simulations in the quasistatic, athermal limit to investigate edge dislocations bowing-out from a row of collinear cylindrical holes. A CAC simulation model, in general, contains an atomistic domain and a coarse-grained domain, in both of which the nucleation and propagation of dislocations and intrinsic stacking faults are permitted with a unified atomistic-continuum integral formulation [25]. In the coarse-grained domain, dislocations can pass between discontinuous elements [26]. Within each element, a finite element method with Gaussian quadrature is used to calculate the force/energy of the integration points and update the nodal positions, from which the positions of atoms inside the element are interpolated [27]. The coarse-grained description yields an accurate generalized stacking fault energy (GSFE) surface because the non-linear dislocation core structure/energy and Burgers vector are naturally accommodated [28].

Previously, CAC was applied to model nucleation and growth of dislocation loops in $\mathrm{Cu}, \mathrm{Al}$, and $\mathrm{Si}$ [29], dislocation-void interactions [30] in $\mathrm{Ni}$, and dislocation-grain boundary interactions in $\mathrm{Cu}$ and $\mathrm{Al}$ [31]. The success of these calculations suggests the viability of using the CAC method to simulate cooperative dislocation bow-out in a sufficiently large model. Focus is placed on the critical shear stress and critical dislocation bow-out configuration - two important characteristics of the dislocation bow-out process. Face-centered cubic (FCC) $\mathrm{Al}$ is chosen because of its high stable SFE $\left(\approx 146 \mathrm{~mJ} / \mathrm{m}^{2}\right)$ and low elastic anisotropy index $(\approx 1.21)$ [32], which enables the simulation results to be compared with most isotropic continuum models that don't consider dislocation dissociation. The embedded-atom method (EAM) potential of Mishin et al. [33] is adopted because the evaluated GSFE 
is close to experimentally measured value [34]. The post-processing follows our earlier work $[28,31]$, where more details of the CAC approach are given. Some runs are completed using Comet and Bridges on the NSF Extreme Science and Engineering Discovery Environment (XSEDE) [35].

Figure 1(a) presents the simulation cell containing a row of 6 collinear cylindrical holes throughout the specimen along the $z$ direction, which would potentially accommodate 5 dislocation segments between them. Full atomistic resolution is applied in the vicinity of holes such that the hole surface is at least $2 \mathrm{~nm}$ from the atomistic/coarse-grained interface; away from the holes, 3D rhombohedral discontinuous elements with surfaces corresponding to $\{111\}$ slip planes are employed [26]. Within each second nearest neighbor element, piecewise continuous first order shape and interpolation functions are used; between elements, neither displacement continuity nor interelement compatibility is required [28]. The lattice orientations are $x[\overline{1} 1 \overline{2}], y[110]$, and $z[1 \overline{1} \overline{1}]$. With the lattice parameter $a_{0}=4.05 \AA$, the simulation cell has a size of $300.59 \mathrm{~nm}$ by $297.22 \mathrm{~nm}$ by $45.49 \mathrm{~nm}$; in all simulations, a uniform hole cross-sectional diameter $D=2.81 \mathrm{~nm}$ is used. With a constant interhole ligament distance $L=5.61 \mathrm{~nm}$, the number of initial dislocation segments $n$ varies from 1 to 14 , corresponding to a total ligament distance $L^{\prime}=n L+(n-1) D$ between the leftmost and rightmost holes of $5.61 \sim 115.07 \mathrm{~nm}$. As a result, the model with only one dislocation segment of $L$ has 108,163 elements and 1,200,322 atoms, with 2,065,626 degrees of freedom in total, compared with $238,834,433$ atoms in an equivalent full atomistic model, which would be highly computationally intensive. Simulations containing only two holes distanced by $L^{\prime}$ are also performed for 
comparison purposes, as shown in Fig. 1(b). All boundaries of the simulation cell as well as the hole surfaces are traction free such that the spurious forces arising from periodic images are eliminated [36]. The image forces on the bow-out from all boundaries can be estimated as follows:

1. In computing the image forces, the image dislocations can be assumed straight [36].

2. The image stresses arising from the surfaces normal to the $x$ axis are stresses of a finite straight dislocation segment. Since the dislocation bow-out is collinear with but not on the image dislocations, the image stresses are zero according to Brown's formula [37].

3. The net image shear stress on the dislocation bow-out from the image dislocations of the two surfaces normal to the $y$ axis is [12]

$$
\tau_{z y}^{\text {image }}=\frac{\mu b}{4 \pi(1-\nu)}\left|\frac{1}{l_{y}^{+}}-\frac{1}{l_{y}^{-}}\right|
$$

where $\mu$ is the shear modulus, $b$ is the magnitude of the Burgers vector of the full dislocation $\mathbf{b}=\left(a_{0} / 2\right)[110], \nu$ is the Poisson's ratio, $l_{y}^{+}$and $l_{y}^{-}$ are the distances between the center-of-mass of the bowed dislocation and the surface whose outward normal vector is along the positive $y$ and negative $y$ directions, respectively. Initially, $l_{y}^{+}=l_{y}^{-}=L_{y} / 2$ where $L_{y}$ is the length of the simulation cell along the $y$ axis; hence, $\tau_{z y}^{\text {image }}=0$. In the extreme case, the dislocations bow out to a semi-elliptic shape such that the center-of-mass of the bowed dislocation is moved by $4 L /(3 \pi)$ from its initial position, resulting in $l_{y}^{+}=148.61-4 L /(3 \pi)=146.23 \mathrm{~nm}$ and $l_{y}^{-}=148.61+4 L /(3 \pi)=150.99 \mathrm{~nm}$. Substituting $\mu=28 \mathrm{GPa}$, $b=0.286 \mathrm{~nm}$, and $\nu=0.33$ into Eq. 1 yields $\tau_{z y}^{\text {image }}=0.205 \mathrm{MPa}$. Note that this net image shear stress promotes dislocation bow-out. 
4. The image forces from the surfaces normal to the $z$ axis don't have any components on the $x-y$ plane, within which the dislocation bowout takes place [12]. Indeed, the net image force is zero since the dislocations are equidistant from both surfaces.

Straight edge dislocations are introduced on the mid-plane normal to the $z$ axis by moving atoms/nodes inside the green lines in Fig. 1 by $\mathbf{b}$, followed by energy minimization. Subsequently, a homogeneous shear stress $\tau_{z y}$ is applied, with atoms in adjacent to the holes constrained within the $x$-y plane to exclude dislocation climb and cross-slip of screw segments; these processes don't accompany the dislocation bow-out under consideration here. We emphasize that in the initial configuration, no dislocation segments pre-exist beyond the leftmost and rightmost holes, unlike those for dislocation/obstacle interactions where an infinitely long dislocation and an infinite array of obstacles are introduced $[38,39]$. The model employed in this work is specifically designed to study the effects of adjacent bowed-out segments on a given dislocation bow-out, as well as whether, in terms of such effects, an increasing, finite number of obstacles would converge to an infinite number of obstacles.

To obtain the critical shear stress and critical dislocation configuration, we conduct quasistatic $\mathrm{CAC}$ simulations at $0 \mathrm{~K}$ with a constant increment of applied shear stress $\Delta \tau_{z y}=1 \mathrm{MPa}$, until the minimum stress for any of the critical events defined below is reached [40]. Two critical events are considered: (I) any dislocation is about to be detached from any hole surface (Fig. 2(a), at $\tau_{z y}^{\mathrm{I}}$ ) and (II) all dislocations are about to be detached from all but the leftmost and rightmost holes (Fig. 2(b), at $\tau_{z y}^{\mathrm{II}}$ ). Clearly, $\tau_{z y}^{\mathrm{I}}<\tau_{z y}^{\mathrm{II}}$. In simulations containing only one dislocation segment of length $L^{\prime}$, as shown in 
Fig. 1(b), a critical event III is considered for which the bowed-out dislocation segment pinned on two holes continues growing until reaching the unstable overall semi-elliptic configuration (Fig. 2(c), at $\tau_{z y}^{\mathrm{III}}$ ). For each critical event, the stress $\tau_{\mathrm{c}}^{k}=\tau_{z y}^{k}-\Delta \tau_{z y}(k=\mathrm{I}$, II, or III) is taken as the critical shear stress [41] and the segment shape at the end of an energy minimization subject to $\tau_{\mathrm{c}}^{k}$ is recorded as the critical dislocation configuration [20]. In particular for the critical event III, in the case of $n=1$, we found that (1) as each element has a larger number of atoms, $\tau_{z y}^{\mathrm{III}}$ becomes smaller, (2) the relative coarsegraining error of $\tau_{z y}^{\mathrm{III}}$ for 2197 atoms per element is approximately 9.5\%, with respect to the fully resolved atomistic simulation.

Shear stresses $\tau_{\mathrm{c}}$ calculated in CAC simulations for critical events I and II are plotted in Fig. 2(d) with respect to the number of initial dislocation segments $n$. The calculated critical stresses $\tau_{\mathrm{c}}^{\mathrm{III}}$ for an isolated dislocation segment with length $L^{\prime}$ to reach the semi-elliptic shape is also shown, where $L^{\prime}$ is determined by $n$ which is taken as the $x$ axis. Also given in Eq. 3(d) are the continuum models for dislocation/obstacle interactions proposed by Scattergood and Bacon [18] and Crone et al. [20], i.e.,

$$
\begin{aligned}
\tau_{\mathrm{SB}} & =\frac{A \mu b}{2 \pi L^{\prime}}\left[\ln \left(\frac{\bar{D}}{r_{0}}\right)+B\right] \\
\tau_{\mathrm{CMK}} & =\frac{A \mu b}{2 \pi\left(L^{\prime}+D / 2\right)} \ln \left(\frac{\bar{D}}{r_{0}}\right)
\end{aligned}
$$

where $A$ and $B$ are parameters, and the dislocation core radius is $r_{0}=b$. For edge dislocations bowing out between holes, $A=1$ and $B=1.52$ [18]. Note that both continuum models assume an infinite row of uniformly spaced obstacles with a ligament distance of $L^{\prime}$ between two neighboring obstacles; in other words, the number of initial dislocation segments $n \rightarrow \infty$. 
For an isolated pair of holes distanced by $L^{\prime}(=L)$, namely, $n=1$, the critical shear stress $\tau_{\mathrm{c}}^{\mathrm{III}}$ lies between the predictions of Eqs. 2 and 3. As $n$ increases, both $\tau_{\mathrm{c}}^{\mathrm{I}}$ and $\tau_{\mathrm{c}}^{\mathrm{II}}$ decrease; for each $n>1$ (equivalently, $L^{\prime}>$ $5.61 \mathrm{~nm})$, there is $\tau_{\mathrm{c}}^{\mathrm{II}}>\tau_{\mathrm{c}}^{\mathrm{I}}>\tau_{\mathrm{c}}^{\mathrm{III}}$. This suggests that as long as dislocations are detached from all holes except the leftmost and rightmost ones (i.e., corresponding to the critical event II), they can always grow beyond the unstable semi-elliptic configuration. For the largest $n$ of $14, \tau_{\mathrm{c}}^{\mathrm{I}}$ and $\tau_{\mathrm{c}}^{\mathrm{II}}$ are $356 \mathrm{MPa}$ and $382 \mathrm{MPa}$, respectively. Note that the relative errors arising from the image stresses are $0.205 / 356=0.057 \%$ and $0.205 / 382=0.054 \%$, respectively; these are negligible. On the other hand, for the same interhole ligament distance $L^{\prime}(=L)$, the CMK model Eq. 3 (which assumes $n \rightarrow \infty$ ) gives $\tau_{\mathrm{CMK}}=342 \mathrm{MPa}$ marked by the horizontal dotted line in Fig. 2(d). The convergence of $\tau_{\mathrm{c}}^{\mathrm{I}}$ and $\tau_{\mathrm{c}}^{\mathrm{II}}$ to $\tau_{\mathrm{CMK}}$ for a larger $n$ shows that an increasing number of obstacles indeed approaches an infinite array of equally spaced obstacles, as assumed in the continuum models.

We emphasize that direct comparisons between simulations and continuum models should be made carefully. For example, critical shear stresses $\tau_{\mathrm{c}}^{\mathrm{III}}$ calculated by MD simulations [42] are found to fit Eq. 2. In Ref. [42], however, (1) the periodic initial dislocation segments are not immediately adjacent to each other, (2) PBCs are adopted in all directions, and (3) the simulations are dynamical with inertial effects. All these issues potentially result in a higher critical stress compared with the quasistatic continuum models. On the other hand, Eq. 2 is known to overestimate the critical stress because of a number of significant simplifications in the model [20], which is also demonstrated in our simulations in that the critical stresses for an 
isolated pair of obstacles surprisingly agree with Eq. 2 which assumes an infinite array of obstacles. Thus, the agreement with MD simulations doesn't necessarily suggest the accuracy of Eq. 2. In this paper, the above-mentioned issues in MD simulations are circumvented by performing quasistatic CAC simulations using sufficiently large models.

Snapshots of dislocation configurations for the critical events I and II are shown in Fig. 3. Subject to the critical shear stress $\tau_{\mathrm{c}}^{\mathrm{I}}$, a dislocation segment is about to be detached from the $2^{\text {nd }}$ hole from the right in Fig. 3(a); its configuration is similar to an isolated pair of holes at $\tau_{\mathrm{c}}^{\mathrm{III}}\left(\right.$ for $\left.L^{\prime}=L\right)$. As the stress increases to $\tau_{\mathrm{c}}^{\mathrm{II}}$, the dislocation pinned at the leftmost and rightmost holes is about to be detached from the $4^{\text {th }}$ hole from the left in Fig. 3(c). By analyzing the intermediate configurations during energy minimization subject to $\tau_{\mathrm{c}}^{\mathrm{II}}$, we find that dislocations are sequentially detached from holes in a random order, as shown in Fig. 3(b).

We are also interested in exploring whether the presence of the intermediate holes in the middle influences the dislocation behavior following the critical event II. To calculate $\tau_{\mathrm{c}}^{\mathrm{III}}$ in models with collinear obstacles, the applied shear stress is first reduced to $10 \mathrm{MPa}$ from $\tau_{\mathrm{c}}^{\mathrm{II}}$ after dislocations are detached from all intermediate holes; then an increment of shear stress $\Delta \tau_{z y}=1 \mathrm{MPa}$ is added until the critical event III is observed. It is shown that the critical stresses are close to those in the case of an isolated pair of holes distanced by $L^{\prime}$, which may reflect that the intermediate holes don't exert long range stress fields.

The dislocation configurations at $\tau_{\mathrm{c}}^{\mathrm{III}}$, however, are affected by the presence of the intermediate holes, as shown in Fig. 4. The ratio of height $\left(H^{\prime}\right.$ or 
$\left.H^{\prime \prime}\right)$ to length $L^{\prime}$ of the dislocation half-loop is about 0.5 and 0.75 with and without the intermediate holes, respectively. The decrease in the dislocation half-loop height is larger for a larger $n$. In all cases, the coarse-grained domain in $\mathrm{CAC}$ retains one key signature of the bowed-out dislocation that the edge components have a larger stacking fault width than the screw components. The segments are nearly semi-elliptic in shape since a screw component is formed to lower the elastic energy $[6,15]$. Moreover, because of the elastic anisotropy of the Shockley partial dislocations, the dislocation half-loop is asymmetric with respect to the $y$ axis [43]. We remark that the kinks along dislocations have a size on the order of that of an element, which is illustrated as a red parallelogram in Fig. 4. Clearly, element discretization is a source of coarse-graining error in the dislocation line energy.

In this paper, we performed CAC simulations to explore the critical shear stress and critical dislocation configuration in the process of edge dislocations bow-out from a row of collinear, uniformly spaced cylindrical holes in FCC Al. It is demonstrated that (1) CAC simulations can explore cooperative dislocations bow-out in sufficiently large models at the submicron scale, (2) as the number of adjacent bowed-out segments increases, the critical shear stress for dislocation depinning approaches that for an infinite array of collinear obstacles, and (3) for the unstable semi-elliptic dislocation configuration, the presence of intermediate obstacles doesn't influence the critical shear stress, but reduces the dislocation half-loop height.

Future work includes applying CAC to more realistic models with obstacles that are non-equidistant, randomly distributed, or with long range stress fields, e.g., forest dislocations. We remark that if the dislocation continues to 
grow, beyond the critical configuration shown in Fig. 4, it will wrap around and interact with the holes. Such interactions, i.e., void hardening [44], will be addressed in a future study that is focused on work hardening behavior that results from dislocation-void interactions.

\section{Acknowledgment}

These results are based upon work supported by the National Science Foundation as a collaborative effort between Georgia Tech (CMMI-1232878) and University of Florida (CMMI-1233113). Any opinions, findings, and conclusions or recommendations expressed in this material are those of the authors and do not necessarily reflect the views of the National Science Foundation. The work of LX was supported in part by the Department of Energy, Office of Basic Energy Sciences under Award Number DE-SC0006539. The authors thank Dr. Alexander Stukowski for providing the dislocation extraction algorithm code. This work used the Extreme Science and Engineering Discovery Environment (XSEDE), which is supported by National Science Foundation grant number ACI-1053575.

\section{References}

[1] B. A. Szajewski, F. Pavia, W. A. Curtin, Modelling Simul. Mater. Sci. Eng. 23 (2015) 085008. URL: http://stacks. iop.org/0965-0393/23/i=8/a=085008. doi:10.1088/0965-0393/23/8/085008.

[2] F. C. Frank, W. T. Read, Phys. Rev. 79 (1950) 722-723. 
URL: $\quad$ http://link.aps.org/doi/10.1103/PhysRev.79.722. doi:10.1103/PhysRev.79.722.

[3] M. de Koning, W. Cai, V. V. Bulatov, Phys. Rev. Lett. 91 (2003) 025503. URL: http://link.aps.org/doi/10.1103/PhysRevLett.91.025503. doi:10.1103/PhysRevLett.91.025503.

[4] X. Y. Li, W. Yang, Phys. Rev. B 74 (2006) 144108. URL: http://link.aps.org/doi/10.1103/PhysRevB.74.144108. doi:10.1103/PhysRevB .74.144108.

[5] A. A. Benzerga, Y. Brchet, A. Needleman, E. V. d. Giessen, Modelling Simul. Mater. Sci. Eng. $12 \quad$ (2004) 159. URL: http://stacks.iop.org/0965-0393/12/i=1/a=014. doi:10. 1088/0965-0393/12/1/014.

[6] B. Gurrutxaga-Lerma, D. S. Balint, D. Dini, A. P. Sutton, J. Mech. Phys. Solids 84 (2015) 273-292. URL: http://www.sciencedirect.com/science/article/pii/S0022509615300715. doi:10.1016/j.jmps. 2015.08.008.

[7] D. Rodney, Y. Le Bouar, A. Finel, Acta Mater. 51 (2003) 17-30. URL: http://www. sciencedirect.com/science/article/pii/S1359645401003792. doi:10.1016/S1359-6454(01)00379-2.

[8] J. Berry, N. Provatas, J. Rottler, C. W. Sinclair, Phys. Rev. B 89 (2014) 214117. URL: http://link.aps.org/doi/10.1103/PhysRevB.89.214117. doi:10.1103/PhysRevB . 89.214117. 
[9] Y. Xiang, L.-T. Cheng, D. J. Srolovitz, W. E, Acta Mater. 51 (2003) 5499-5518. URL: http://www. sciencedirect.com/science/article/pii/S1359645403004154. doi:10.1016/S1359-6454(03)00415-4.

[10] D. N. Seidman, E. A. Marquis, D. C. Dunand, Acta Mater. 50 (2002) 4021-4035. URL: http://www. sciencedirect. com/science/article/pii/S135964540200201X. doi:10.1016/S1359-6454(02)00201-X.

[11] J. M. Rosalie, H. Somekawa, A. Singh, T. Mukai, J. Alloys Compd. 550 (2013) 114-123. URL: http://www. sciencedirect. com/science/article/pii/S0925838812015952. doi:10.1016/j.jallcom.2012.09.027.

[12] D. Hull, D. J. Bacon, Introduction to Dislocations, 5th ed., ButterworthHeinemann, 2011.

[13] S. S. Shishvan, S. Mohammadi, M. Rahimian, Modelling Simul. Mater. Sci. Eng. 16 (2008) 075002. URL: http://iopscience.iop.org/0965-0393/16/7/075002. doi:10. 1088/0965-0393/16/7/075002.

[14] J. P. Hirth, J. Lothe, Theory of Dislocations, Krieger Pub Co, 1992.

[15] A. J. E. Foreman, Philos. Mag. 15 (1967) 1011-1021. URL: $\quad$ http://dx.doi.org/10.1080/14786436708221645. doi:10.1080/14786436708221645. 
[16] D. J. Bacon, Phys. Stat. Sol. (b) 23 (1967) 527-538. URL: http://onlinelibrary.wiley.com/doi/10.1002/pssb.19670230212/abstract. doi:10.1002/pssb. 19670230212.

[17] D. J. Bacon, U. F. Kocks, R. O. Scattergood, Philos. Mag. 28 (1973) 1241-1263. URL: http://dx.doi.org/10.1080/14786437308227997. doi:10.1080/14786437308227997.

[18] R. O. Scattergood, D. J. Bacon, Acta Metall. 30 (1982) 1665-1677. URL: http://www.sciencedirect.com/science/article/pii/0001616082901882. doi:10.1016/0001-6160(82) 90188-2.

[19] J. Friedel, Dislocations, Pergamon Press, 1964.

[20] J. C. Crone, L. B. Munday, J. Knap, Acta Mater. 101 (2015) 40-47. URL: http://www.sciencedirect. com/science/article/pii/S1359645415006540. doi:10.1016/j. actamat.2015.08.067.

[21] A. J. E. Foreman, M. J. Makin, Philos. Mag. 14 (1966) 911-924. URL: http://dx.doi.org/10.1080/14786436608244762. doi:10.1080/14786436608244762.

[22] U. F. Kocks, Can. J. Phys. 45 (1967) 737-755. URL: http://www.nrcresearchpress.com/doi/abs/10.1139/p67-056. doi:10.1139/p67-056.

[23] T. Nogaret, D. Rodney, Phys. Rev. B 74 (2006) 134110. URL: http://link.aps.org/doi/10.1103/PhysRevB.74.134110. doi:10.1103/PhysRevB.74.134110. 
[24] C. Sobie, N. Bertin, L. Capolungo, Metall. Mater. Trans. A 46 (2015) 3761-3772. URL: http://link. springer.com/article/10.1007/s11661-015-2935-z. doi:10.1007/s11661-015-2935-z.

[25] Y. Chen, J. Chem. Phys. 130 (2009) 134706. URL: http://jcp.aip.org/resource/1/jcpsa6/v130/i13/p134706_s1. doi:doi:10.1063/1.3103887.

[26] L. Xiong, G. Tucker, D. L. McDowell, Y. Chen, J. Mech. Phys. Solids 59 (2011) 160-177. URL: http://www. sciencedirect. com/science/article/pii/S0022509610002395. doi:10.1016/j.jmps. 2010.11.005.

[27] S. Xu, L. Xiong, Q. Deng, D. L. McDowell (2016).

[28] S. Xu, R. Che, L. Xiong, Y. Chen, D. L. McDowell, Int. J. Plast. 72 (2015) 91-126. URL: http://www.sciencedirect.com/science/article/pii/S0749641915000777. doi:10.1016/j.ijplas.2015.05.007.

[29] L. Xiong, D. L. McDowell, Y. Chen, Scripta Mater. 67 (2012) 633-636. URL: http://www.sciencedirect.com/science/article/pii/S1359646212004691. doi:10.1016/j.scriptamat.2012.07.026.

[30] L. Xiong, S. Xu, D. L. McDowell, Y. Chen, Int. J. Plast. 65 (2015) 33-42. URL: 
http://www. sciencedirect. com/science/article/pii/S0749641914001508. doi:10.1016/j.ijplas.2014.08.002.

[31] S. Xu, L. Xiong, Y. Chen, D. L. McDowell, npj Comput. Mater. 2 (2016) 15016. URL: http://www . nature. com/articles/npjcompumats201516. doi:10.1038/npj compumats. 2015.16.

[32] K. Ramesh, Nanomaterials: Mechanics and Mechanisms, Springer, New York, 2009.

[33] Y. Mishin, D. Farkas, M. J. Mehl, D. A. Papaconstantopoulos, Phys. Rev. B 59 (1999) 3393-3407. URL: http://link.aps.org/doi/10.1103/PhysRevB.59.3393. doi:10.1103/PhysRevB.59.3393.

[34] M. Chassagne, M. Legros, D. Rodney, Acta Mater. 59 (2011) 1456-1463. URL: http://www.sciencedirect.com/science/article/pii/S1359645410007512. doi:10.1016/j. actamat.2010.11.007.

[35] J. Towns, T. Cockerill, M. Dahan, I. Foster, K. Gaither, A. Grimshaw, V. Hazlewood, S. Lathrop, D. Lifka, G. Peterson, R. Roskies, J. Scott, N. Wilkins-Diehr, Comput. Sci. Eng. 16 (2014) 62-74. doi:10.1109/MCSE. 2014.80.

[36] B. A. Szajewski, W. A. Curtin, Modelling Simul. Mater. Sci. Eng. 23 (2015) 025008. URL: 
[37] L. M. Brown, Philos. Mag. $15 \quad$ (1967) 363-370. URL: $\quad$ http://dx.doi.org/10.1080/14786436708227708. doi:10.1080/14786436708227708.

[38] G. Monnet, Acta Mater. 55 (2007) 5081-5088. URL: http://www.sciencedirect.com/science/article/pii/S135964540700359X. doi:10.1016/j.actamat. 2007.05.030.

[39] Y. N. Osetsky, D. J. Bacon, Modelling Simul. Mater. Sci. Eng. 11 (2003) 427-446. URL: http://stacks.iop.org/0965-0393/11/i=4/a=302. doi:10 . 1088/0965-0393/11/4/302.

[40] T. Ohashi, M. Kawamukai, H. Zbib, Int. J. Plast. 23 (2007) 897-914. URL: http://www.sciencedirect.com/science/article/pii/S074964190600129X. doi:10.1016/j.ijplas.2006.10.002.

[41] S. G. Srinivasan, X. Z. Liao, M. I. Baskes, R. J. McCabe, Y. H. Zhao, Y. T. Zhu, Phys. Rev. Lett. 94 (2005) 125502. URL: http://link.aps.org/doi/10.1103/PhysRevLett.94.125502. doi:10.1103/PhysRevLett.94.125502.

[42] T. Shimokawa, S. Kitada, Mater. Trans. 55 (2014) 58-63. doi:10.2320/matertrans. MA201319.

[43] M.-S. Huang, Y.-X. Zhu, Z.-H. Li, Chin. Phys. Lett. 31 (2014) 
046102. URL: http://iopscience.iop.org/0256-307X/31/4/046102. doi:10.1088/0256-307X/31/4/046102.

[44] T. A. Khraishi, H. M. Zbib, T. D. d. l. Rubia, M. Victoria, Metall. Mater. Trans. B 33 (2002) 285-296. URL: http://link. springer. com/article/10.1007/s11663-002-0012-7. doi:10.1007/s11663-002-0012-7.

[45] A. Stukowski, V. V. Bulatov, A. Arsenlis, Modelling Simul. Mater. Sci. Eng. 20 (2012) 085007. URL: http://iopscience.iop.org/0965-0393/20/8/085007/cites. doi:10.1088/0965-0393/20/8/085007. 


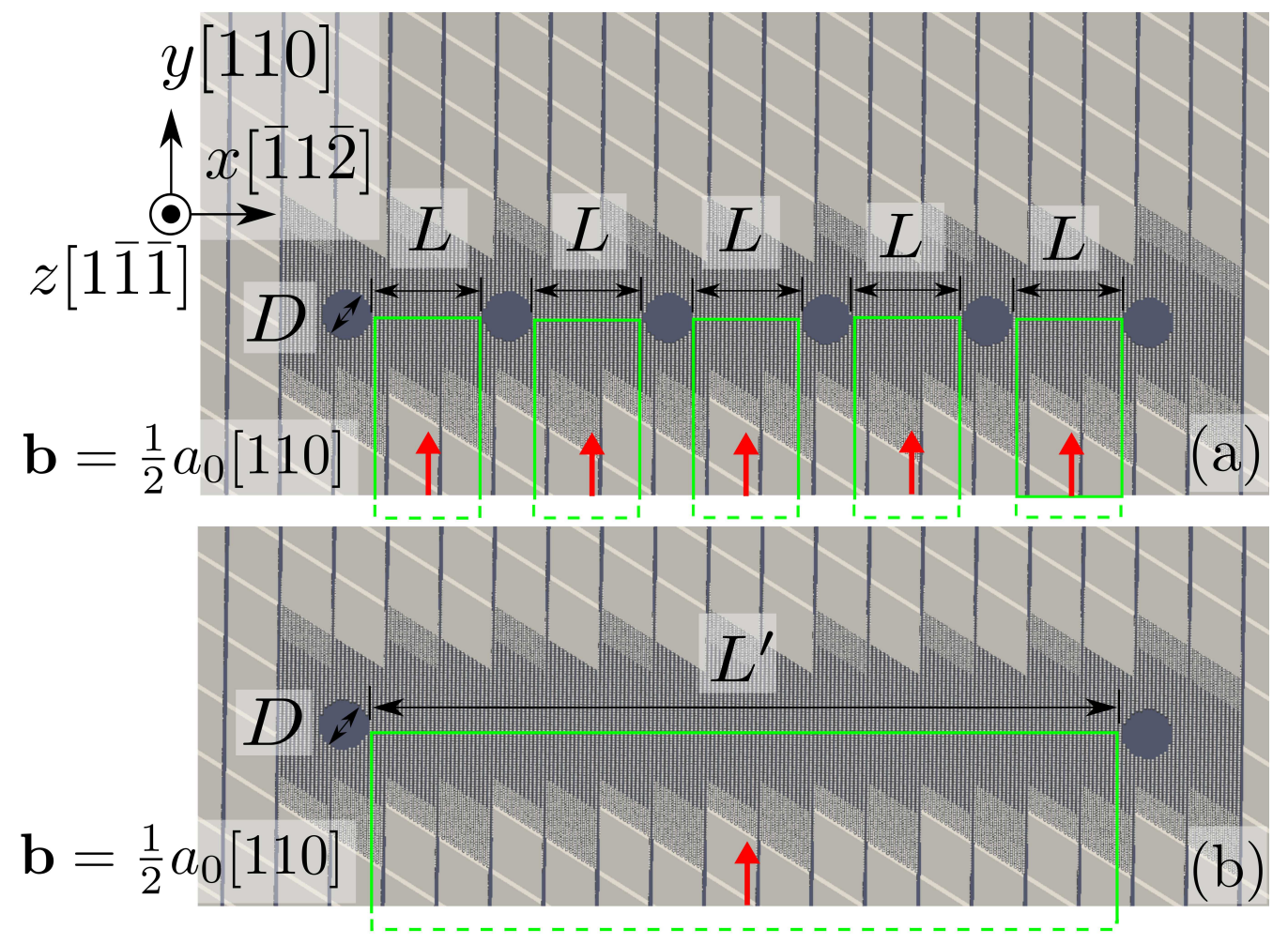

Figure 1: CAC simulation cell containing (a) a row of 5 collinear initial dislocation segments each of which has a length of $L$ and (b) one initial dislocation segment with length $L^{\prime}=5 L+4 D$, where $L=5.61 \mathrm{~nm}$ and $D=2.81 \mathrm{~nm}$. Cylindrical holes are throughout the specimen along the $z$ direction. An atomistic domain is retained in the vicinity of the holes, while the coarse-grained domain is employed elsewhere. All boundaries are assumed stress free to alleviate spurious periodic image forces. Edge dislocations are formed by moving atoms/nodes inside the green lines by Burgers vector $\mathbf{b}=\left(a_{0} / 2\right)[110]$. 


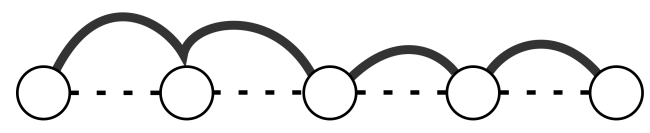

(a) Critical event I

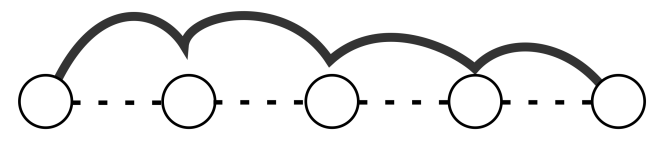

(b) Critical event II

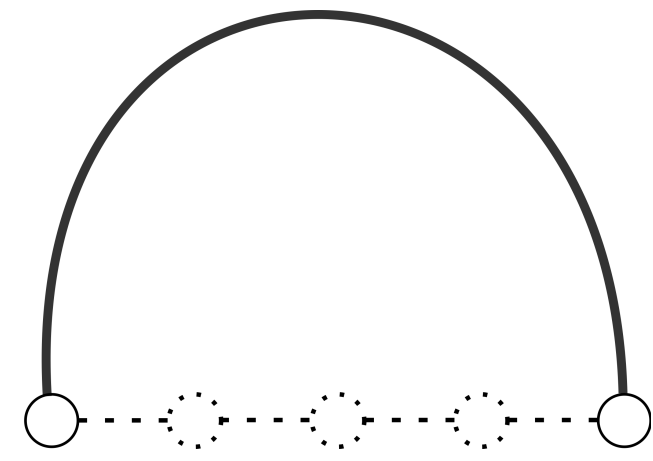

(c) Critical event III

Total ligament distance $L(\mathrm{~nm})$

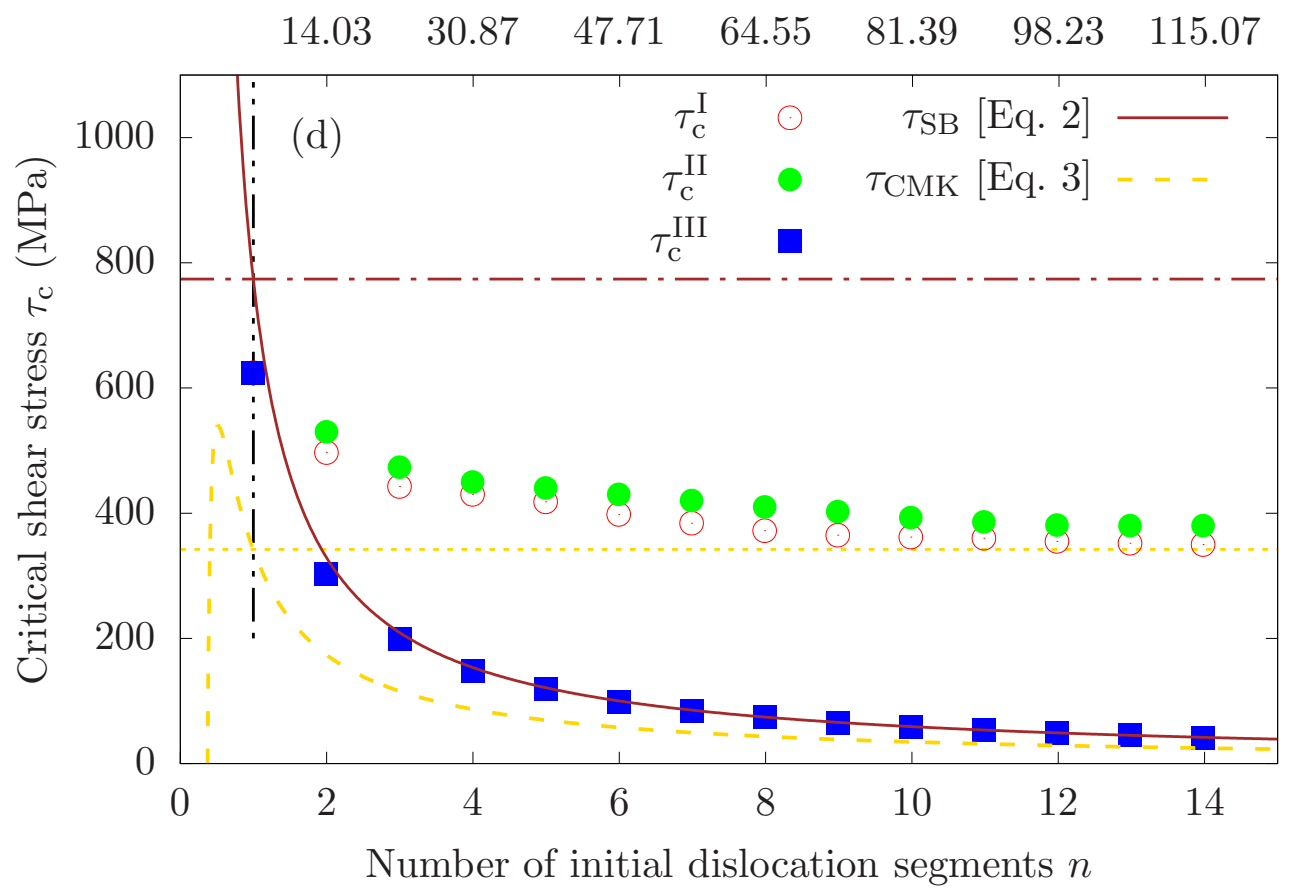

Figure 2: $(\mathrm{a}-\mathrm{c})$ Dislocation configurations at the three critical events studied in this work; (d) shear stresses $\tau_{\mathrm{c}}$ calculated in CAC simulations for three critical events, with respect to the number of initial dislocation segments $n$. Critical shear stresses for one dislocation segment with length $L^{\prime}$ predicted by continuum models Eqs. 2 and 3 are also shown. The horizontal dash-dot and dotted lines are the critical shear stresses predicted by Eq. 2 and Eq. 3, respectively, for $L^{\prime}=5.61 \mathrm{~nm}$. 


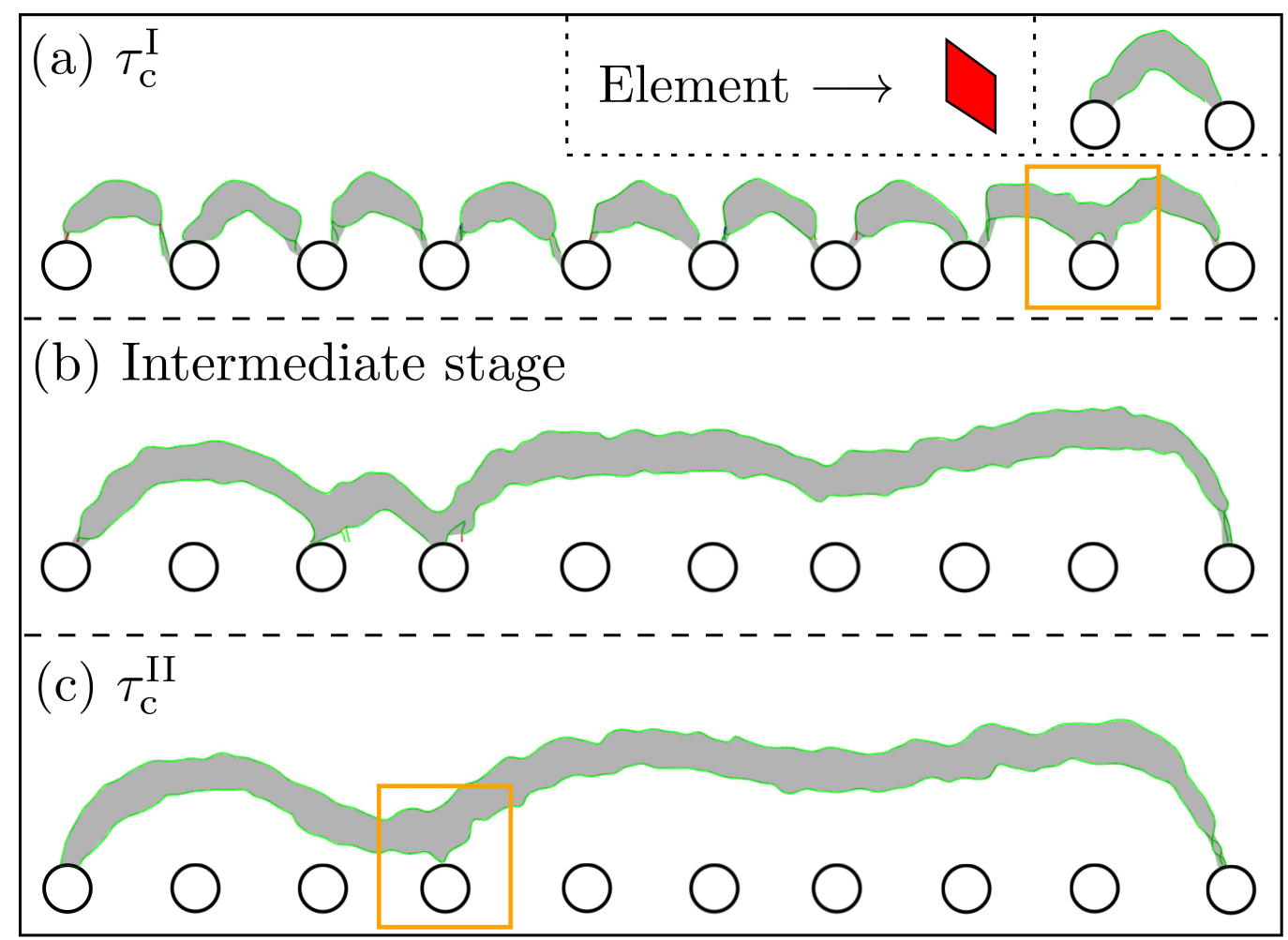

Figure 3: Snapshots of dislocation configurations at the critical events I and II, as well as an intermediate stage. The green curves represent Shockley partial dislocations identified by a Dislocation Extraction Algorithm [45]; the gray ribbons are intrinsic stacking faults. In (a) and (c), dislocations are about to be detached from the holes marked by solid brown lines. The critical dislocation configuration for an isolated pair of holes distanced by $L^{\prime}(=L)$ at $\tau_{\mathrm{c}}^{\mathrm{III}}$ is given at the top right corner of (a). The kinks along dislocations have a size on the order of that of an element, which is illustrated as a red parallelogram. 


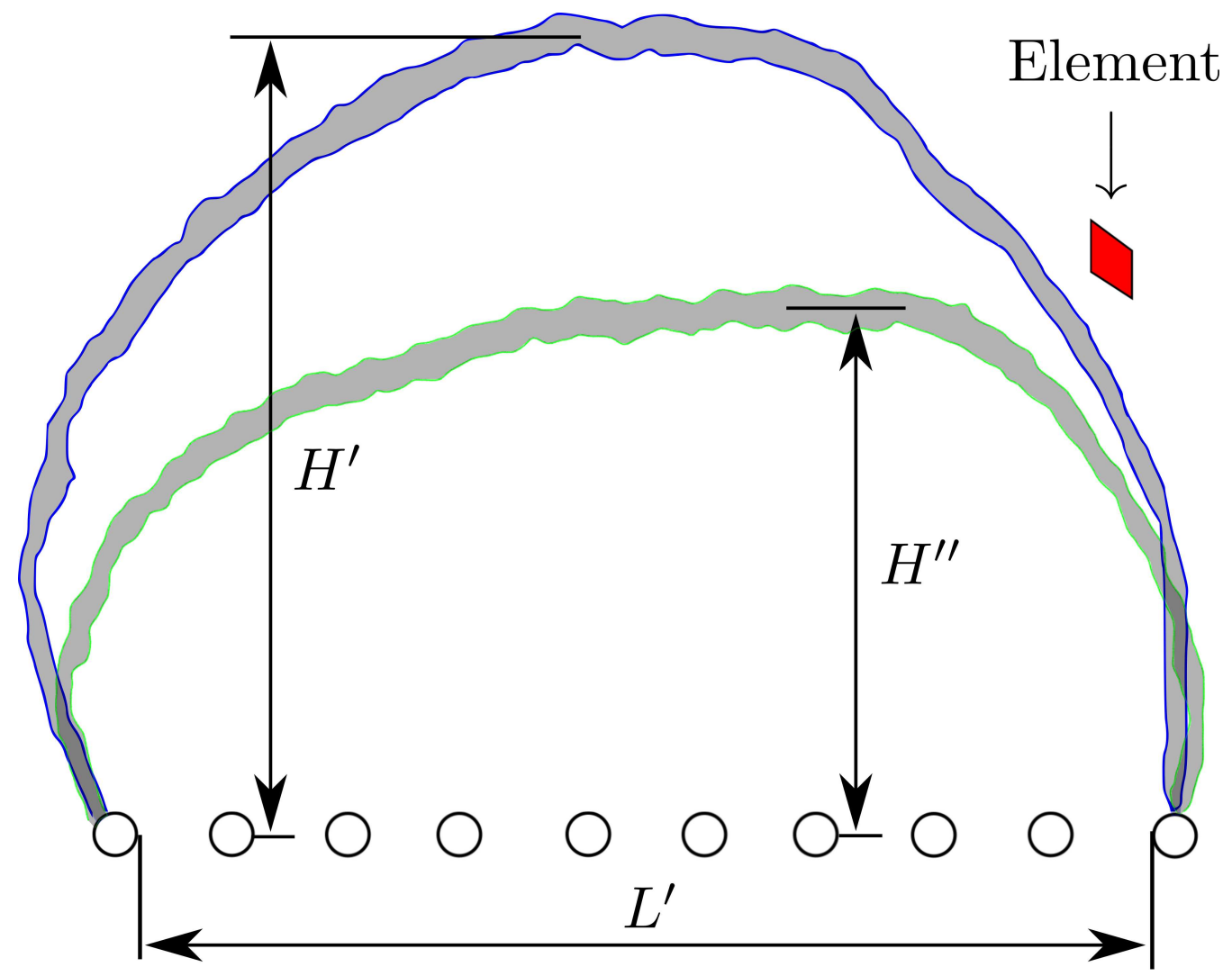

Figure 4: Snapshots of dislocation configurations at the critical event III in the case of 9 adjacent initial dislocation segments (leading and trailing Shockley partials are shown in green) and the case of 1 isolated initial segment with length $L^{\prime}$ (both partials are in blue). Dislocations and stacking faults are identified in the same way as in Fig. 3. The dislocation half-loop heights $H^{\prime}$ and $H^{\prime \prime}$ are about $0.75 L^{\prime}$ and $0.5 L^{\prime}$, respectively. 
(a) $\tau_{\mathrm{c}}^{\mathrm{I}}$

Element $\longrightarrow$
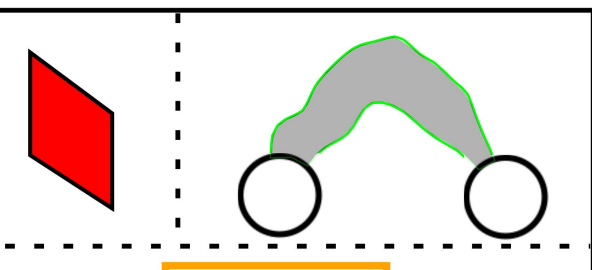

0600000

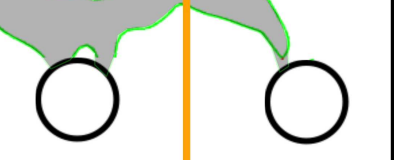

(b) Intermediate stage

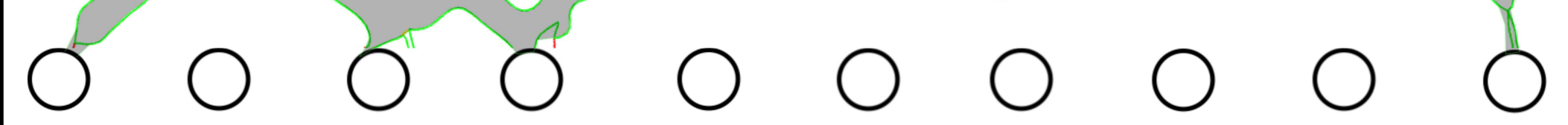

\footnotetext{
(c) $\tau_{\mathrm{c}}^{\mathrm{II}}$
}
00
$\bigcirc 0$
O
O
O
O 